be observed in space, but smaller objects can fall undetected or disintegrate in the atmosphere, whereas they would leave a mark on the Moon.

The crater count could also lead to a recalibration of methods for estimating the age of surfaces elsewhere in the Solar System. Right now, the Moon acts as a sort of fundamental clock. Scientists have dated lunar samples returned to Earth by Apollo and linked those dates to the crater density of the sample's original terrain. So when a surface with a certain crater density is found on Mars, for example, researchers compare it with surfaces on the Moon to pin down its age.

However, corrections must be applied, owing to differences in impact rates between the Moon and Mars. These are estimated from asteroid orbit calculations, Mars's location in the Solar System and models that account for its greater size and gravity.

By combining LRO observations with those from other spacecraft, scientists may be able to determine relative impact rates throughout the Solar System more directly. McEwen and his team have been finding new craters on Mars for the past four years, using data from NASA's Mars Reconnaissance Orbiter. And a current impact rate for Mercury may emerge when NASA's MESSENGER mission begins to orbit the planet next year, although McEwen says that new craters would have to be very large to be detected.

Schultz says that this is an opportunity to improve the dating of surfaces on other planets with measurements rather than models. "You want to see what nature shows you," he says. Roberta Kwok

But David Cane, a biochemist at Brown University in Providence, Rhode Island, believes that a single chip capable of monitoring all the enzymes in a cell is not currently feasible.

He points out that enzymes are picky about the shapes of their preferred substrates. In the reactome array, substrates are attached to a dye molecule and to a linker holding them to the chip, which may change their shape enough to stop them fitting into an enzyme's active site. Cane says that the paper presents no evidence that the assay would work with choosy enzymes.

Ronald Frank of the Helmholtz centre, who coordinated the German investigation, says that a meeting will be held on 11 August to discuss whether there should be further consequences for scientists there. A spokesperson for Science says that the journal is in discussions with some of the institutions involved and will make a decision about the paper "very soon".

Alison Abbott

Additional reporting by Daniel Cressey

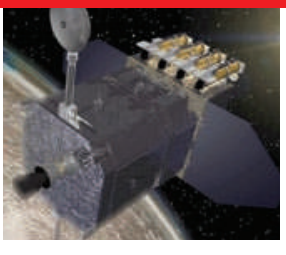

SOLAR FLARES PROBED

First results from Solar Dynamics Observatory go.nature.com/PM5zEg

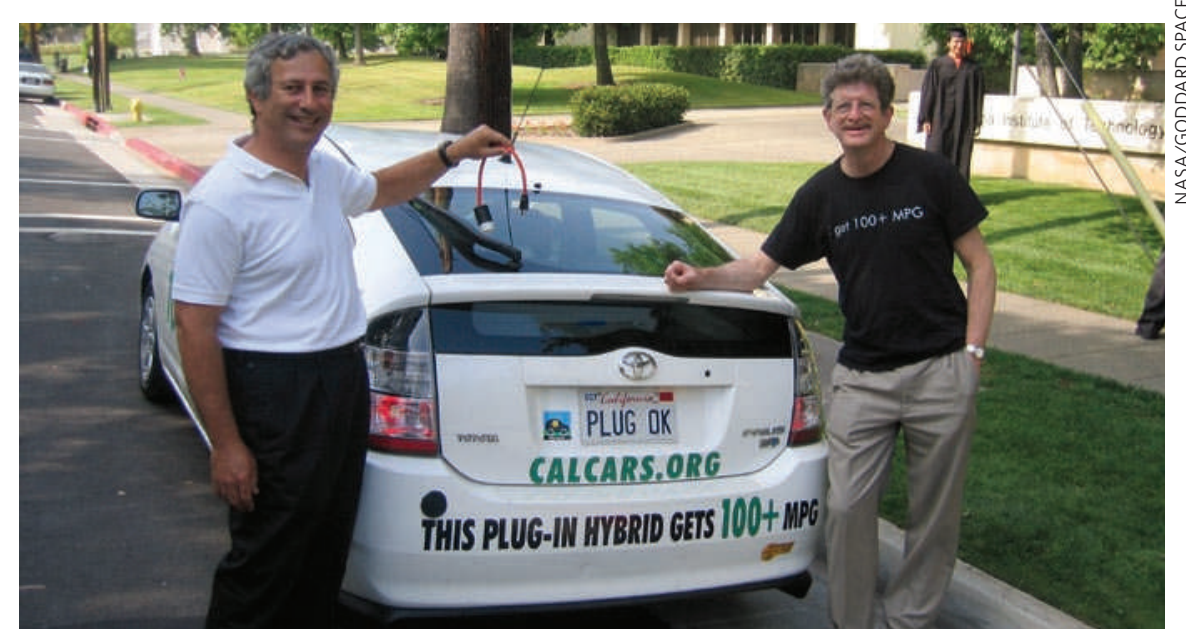

Nate Lewis (left) will direct a \$122-million research project to make fuel directly from sunlight.

\title{
US seeks solar flair for fuels
}

The US Department of Energy has launched an 'artificial photosynthesis' initiative with the ambitious goal of developing, scaling up and ultimately commercializing technologies that directly convert sunlight into hydrogen and other fuels.

The Joint Center for Artificial Photosynthesis (JCAP) will receive US\$122 million over five years, and will be jointly led by the California Institute of Technology (Caltech) in Pasadena and the Lawrence Berkeley National Laboratory in Berkeley, California. Announced on 22 July, JCAP is the second of three Energy Innovation Hubs that US energy secretary Steven Chu plans to establish this year.

The hubs aim to get "We have to scale up from the nanoscale to the macroscale." basic science out of the lab and into the real world. "We have to scale up from the nanoscale to the macroscale," says Nate Lewis, a Caltech chemist who will direct the JCAP programme, which will ultimately employ 150-200 people in two buildings at Caltech and the Berkeley lab.

Existing photovoltaic cells capture photons and produce electricity, which can be used to split water molecules and produce hydrogen. With artificial photosynthesis, photons from the Sun would drive a 'wireless' chemical conversion process to generate fuels. The most likely fuel is hydrogen, which can be used as it is or converted into other liquid fuels as a replacement for petroleum.

Caltech and the Massachusetts Institute of Technology in Cambridge are already leading a similar effort, known as 'Powering the Planet', that was funded with a \$20-million, 5-year grant from the National Science Foundation in 2008. But Lewis says that project focuses more on basic science questions at each step of the process, such as how to boost the number of photons captured by a given material or increase the efficiency of a given catalyst.

By contrast, JCAP aims from the outset to build a solar-fuel system that will ultimately prove commercially viable. Potential advances in electrolysis and photovoltaic solar panels would be welcome but incidental, says Lewis. "That's the dividing line that we drew intellectually." Lewis adds that the centre will compare materials and processes used by competing researchers to determine which scientific avenues look most promising.

Chu initially proposed eight energy hubs, but Congress authorized the energy department to move forward with three. The first energy hub, which focuses on advanced nuclear reactors, was announced in May. A third hub aimed at energyefficient buildings will follow in the coming months. Chu has called the energy hubs 'Bell lablets' after the famous Bell Laboratories in Murray Hill, New Jersey.

Earlier this year in its fiscal 2011 budget, the energy department requested \$34 million for a fourth hub, focusing on batteries and electricity storage, but Congress has yet to approve it. Jeff Tollefson 